\title{
Improved fractional step method for simulating fluid-structure interaction using the PFEM
}

\author{
Minjie Zhu and Michael H. Scott ${ }^{*, \dagger}$ \\ School of Civil and Construction Engineering, Oregon State University, Corvallis, OR, 97331, USA
}

\begin{abstract}
SUMMARY
Numerical difficulties are present in the particle finite element method even though it has been shown to be a powerful and effective approach to simulating fluid-structure interaction. To overcome problems of mass loss on the free surface and the added-mass effect, an improved fractional step method (FSM) that handles addedmass terms in a mathematically exact way is developed. A further benefit is that no assumptions regarding the structural response are made in handling added-mass terms, thus it is straightforward to incorporate material nonlinearity in fluid-structure interaction (FSI) under this approach. Patch tests and comparisons with experimental data are presented in order to verify and validate the improved FSM for FSI applications. The computational cost of this approach is shown to be negligible compared with the other aspects of the FSM, particularly when the size of the structure and the fluid-structure interface is small relative to the volume of fluid. Copyright $\odot 2014$ John Wiley \& Sons, Ltd.
\end{abstract}

Received 14 February 2014; Revised 23 May 2014; Accepted 26 May 2014

KEY WORDS: finite element methods; fluid-structure interaction; incompressible flow; Lagrangian; particle methods

\section{INTRODUCTION}

As structural design codes evolve, for example, in response to the 2011 Tohoku earthquake and tsunami, in order to safeguard coastal infrastructure against tsunami-induced wave and debris loads, the prediction of structural response to these loads is of paramount importance. As a result, accurate and efficient simulation models for fluid-structure interaction (FSI) are required in order to simulate the laboratory experiments and field observations that will drive design code changes. A variety of simulation approaches have been developed for fluid-structure interaction with incompressible Newtonian flows using either the Lagrangian, Eulerian, or Arbitrary Lagrangian-Eulerian formulations [1-5]. Although the Eulerian and Arbitrary Lagrangian-Eulerian formulations have their advantages, important advantages of Lagrangian formulations for FSI simulation of engineered coastal infrastructure include easy tracking of the fluid-free surface and conformity with the Lagrangian formulation of structural mechanics.

The particle finite element method (PFEM), originally proposed by [6], has been shown to be an effective Lagrangian approach to FSI. The PFEM uses a computational procedure that is analogous to that used in finite element analysis of solids, thereby alleviating the need for awkward coupling of disparate numerical modules in FSI simulations. The fractional step method (FSM) is used by the PFEM in order to solve the monolithic system of pressure and momentum equations. In its original form, the FSM splits the pressure from the momentum equations then solves for the pressure using

\footnotetext{
*Correspondence to: Michael H. Scott, Oregon State University, School of Civil and Construction Engineering, 101 Kearney Hall, Corvallis, OR, 97331, USA.

†E-mail: michael.scott@oregonstate.edu 
a continuous Laplace matrix, which is an approximation of the exact pressure tangent matrix [6]. This required pressures to be imposed at the free surface as Dirichlet boundary conditions, which was later found to be responsible for the mass loss at the free surface [7, 8]. Furthermore, interaction between fluid and structure is calculated by a staggered scheme, which will become divergent if the 'added-mass effect' is strong. The added-mass effect is a numerical instability that can lead to nonconvergence of FSI simulations where the fluid and solid have similar densities, the structure is very flexible, or the simulation time steps are very small [9]. As a result, the classic FSM is well-suited for simulations of fluid interacting with rigid structures.

The problem of mass loss on the free surface can be mitigated by an FSM with incompressibility (FSMI) on the free-surface [8]. The FSMI assumes a compressibility coefficient for fluid near the free-surface so that a new boundary term on the free-surface is introduced in order to avoid singularity of the continuous Laplace matrix. However, the compressibility is fictitious, and the choice of the compressibility coefficient is problem dependent.

To avoid imposing problem-dependent pressure boundary conditions, an algebraic splitting scheme can be applied directly to the monolithic system of discretized [7]. The resulting tangent matrix for pressure, called the Pressure Schur Complement (PSC), replaces the continuous Laplace matrix, which is an approximation of the PSC. The algebraic splitting scheme is also called 'FSM with discrete Laplace matrix' [8], as opposed to the continuous Laplace matrix in the classical FSM. The FSM with discrete Laplace matrix works well in solving the problem of mass loss for fluid only problems; however, it becomes intractable for fluid-structure interaction because it requires the inverse of a general structural tangent matrix. This method also suffers from numerical divergence when the added-mass effect is strong because it uses a staggered scheme for fluid-structure interaction.

To overcome the added-mass effect for FSI problems, [9] proposed an interface Laplace matrix that adds mass and stiffness to the system of equations. With this interface Laplace matrix approach, convergent solutions can be obtained when the added-mass effect is strong, and the convergence rate is generally improved for problems where the effect is less significant. Even though the added-mass effect is mitigated, a significant limitation of this approach is its restriction to elastic structures. This can be important when attempting to simulate structural damage and/or collapse due to wave and debris loading.

The objective of this paper is to develop the FSM with discrete Laplace matrix, or PSC, where the exact added-mass terms are retained and handled in the general algebraic monolithic system of FSI in a mathematically exact way. Both mass loss and the added-mass effect, which are caused by approximation of the aforementioned terms, are mitigated to a significant degree, giving virtually no mass loss and very fast convergence when compared with previous versions of the FSM. This comes with the additional expense of computing the 'exact' PSC, which requires the inverse of a general sparse matrix and two matrix-matrix multiplications. However, it will be shown that by utilizing the structure of the monolithic system of algebraic equations for FSI, the cost of matrix inversion is only related to the size of the structure and the fluid-structure interface, instead of the size of the entire problem. For most realistic FSI applications, the size of the structure and the fluid-structure interface is very small compared with the overall problem size. An additional, and perhaps more attractive, advantage of the exact method is that no assumption is made towards the constitutive response of the structure. As a result, the fluid is able to interact with material nonlinear structures using the exact added-mass terms without any additional difficulties.

\section{GOVERNING EQUATIONS OF THE PFEM}

Like other FEMs, the PFEM discretizes the governing equations of FSI in to a system of linear algebraic equations. Differential equations of linear momentum describe the structural response, whereas for incompressible flow and additional mass conservation, equations constrain the fluid velocities to be divergence free. The constitutive equations relate the strain in structures and the stain rate in fluids to stresses. 


\subsection{Differential momentum equations}

For both the fluid and structural domains, the differential equation for conservation of linear momentum is

$$
\rho \dot{v}_{i}=\frac{\partial \sigma_{i j}}{\partial x_{j}}+\rho b_{i}
$$

where $v_{i}$ is the velocity vector, $\sigma_{i j}$ is the Cauchy stress tensor, $x_{j}$ is the current position vector, $b_{i}$ is the body force vector, and $\rho$ is the density. Neumann boundary conditions for both domains are enforced for prescribed normal stresses on the surface, $\Gamma_{t}$,

$$
\sigma_{i j} n_{j}=t_{i}
$$

where $t_{i}$ is the surface traction and $n_{j}$ is the unit normal vector to the boundary surface. The Dirichlet boundary conditions and initial conditions are imposed on displacements or velocities

$$
u_{i}=u_{i}^{p} \quad u_{i}=u_{i}^{0}, \quad v_{i}=v_{i}^{p} \quad v_{i}=v_{i}^{0}
$$

where $u_{i}^{p}$ and $v_{i}^{p}$ are the fixed displacement, and velocity on the boundary and $u_{i}^{0}$ and $v_{i}^{0}$ are the initial displacement and velocity, respectively. The current coordinates of each point in the structural and fluid can be calculated by

$$
x_{i}=x_{i}^{0}+u_{i}
$$

where $x_{i}^{0}$ is the initial position.

\subsection{Mass conservation equations}

Conservation of mass is satisfied in the structural domain by construction, whereas for incompressible flow in the fluid domain, mass conservation can be expressed as the divergence of the velocity field equal to zero

$$
\frac{\partial v_{i}}{\partial x_{i}}=0
$$

\subsection{Constitutive equations}

To consider arbitrary structures in the interaction with fluid, a general stress-strain relationship is used, encompassing both linear and nonlinear, elastic, and inelastic, constitutive models

$$
\sigma_{i j} \equiv \sigma_{i j}\left(\varepsilon_{k l}\right)
$$

where $\varepsilon_{k l}$ is the strain tensor computed from derivatives of the displacement field,

$$
\varepsilon_{k l}=\frac{1}{2}\left(\frac{\partial u_{k}}{\partial x_{l}}+\frac{\partial u_{l}}{\partial x_{k}}\right)
$$

Newtonian fluid flows are assumed, for which the constitutive equations are

$$
\sigma_{i j}=S_{i j}-p \delta_{i j}
$$

where $\delta_{i j}$ is the Kronecker delta and $p$ is the fluid pressure. The deviatoric stress tensor, $S_{i j}$, is related to the strain rate $\dot{\varepsilon}_{i j}$ in the fluid by the viscosity $\mu$.

$$
S_{i j}=2 \mu \dot{\varepsilon}_{i j}
$$




\section{SHAPE FUNCTIONS FOR FINITE ELEMENT DISCRETIZATION}

Using the particle velocity, $v_{i}$, and pressure, $p$, as primary unknowns, the standard Galerkin weighted residual method is applied to the differential momentum equations (1), mass conservation equations (5), and boundary conditions,

$$
\begin{gathered}
\int_{V} \delta v_{i}\left(\rho \dot{v}_{i}-\frac{\partial \sigma_{i j}}{\partial x_{j}}-\rho b_{i}\right) d V-\int_{\Gamma_{t}} \delta v_{i} t_{i} d \Gamma_{t}=0 \\
\int_{V} q \frac{\partial v_{i}}{\partial x_{i}} d V=0
\end{gathered}
$$

where $\delta v_{i}$ and $q$ are weighting functions that satisfy the Dirichlet boundary conditions. The velocity and pressure are approximated in the finite element space using shape functions [10],

$$
v_{i}=\sum_{j=1}^{n_{v}} N_{v}^{j} v_{i}^{j} \quad p=\sum_{j=1}^{n_{p}} N_{p}^{j} p^{j}
$$

where $n_{v}$ and $n_{p}$ is the number of velocity and pressure nodes, respectively. $N_{v}^{j}$ and $N_{p}^{j}$ are the shape functions for velocity and pressure. Suitable choices of shape functions for incompressible fluid conditions are listed in [10]. Because Lagrangian formulation is used in the PFEM, triangular elements for planar analysis and tetrahedral elements for three dimensional analysis are better choices than quadrilaterals for the fluid domain due the large displacements of fluid nodes.

\section{DISCRETE ALGEBRAIC EQUATIONS}

\subsection{Discrete fluid equations}

Through standard finite element procedures, the assembled algebraic equations for the fluid domain are

$$
\begin{gathered}
\mathbf{M}_{f} \dot{\mathbf{v}}_{f}+\mathbf{K}_{f} \mathbf{v}_{f}-\mathbf{G}_{f} \mathbf{p}=\mathbf{F}_{f} \\
\mathbf{G}_{f}^{T} \mathbf{v}_{f}=\mathbf{0}
\end{gathered}
$$

where $\mathbf{v}_{f}$ and $\mathbf{p}$ are velocity and pressure vectors of fluid particles; $\mathbf{F}_{f}$ is the vector of external forces; $\mathbf{M}_{f}$ is the fluid mass matrix; $\mathbf{K}_{f}$ represents the viscosity of the fluid, which is similar to the stiffness matrix in the structure. The matrix $\mathbf{G}_{f}$ is the gradient operator of pressures. Further information on these matrices is found in [11].

Equations (13) and (14) should be stabilized in order to satisfy the Inf-Sup condition for the velocity/pressure formulation. However, the discussion in this paper is independent of the stabilization method chosen. Generally, a stabilized mass equation has the form of

$$
\mathbf{G}_{f}^{T} \mathbf{v}_{f}+\mathbf{S p}=\mathbf{F}_{p}
$$

where $\mathbf{S}$ is the stabilization matrix and $\mathbf{F}_{p}$ is the corresponding right-hand side vector. For example, the $\mathbf{S}$ and $\mathbf{F}_{p}$ for the classic PFEM using finite calculus method [11] are

$$
\begin{gathered}
\mathbf{S}=\mathbf{L} \\
\mathbf{F}_{p}=-\mathbf{Q}^{-1} \mathbf{Q}^{T} \mathbf{p}^{j}
\end{gathered}
$$


where $\mathbf{L}$ is the Laplacian operator; $\mathbf{Q}$ and $\hat{\mathbf{M}}$ are stabilization matrices; and $\mathbf{p}^{j}$ is the pressure vector of the last iteration in a time step. For stable elements that satisfy the Inf-Sup condition for incompressible fluid [12], $\mathbf{S}$ and $\mathbf{F}_{p}$ are both equal to zero, due to the fact that the pressure has been stabilized by the mixed finite element formulations.

$$
\begin{gathered}
\mathbf{S}=\mathbf{0} \\
\mathbf{F}_{p}=\mathbf{0}
\end{gathered}
$$

For stable elements with a bubble mode [13], static condensation within the element will cause $\mathbf{S}$ and $\mathbf{F}_{p}$ to become non-zero. The equivalence between the bubble function and stabilization formulation has been proved in the literature [14-16].

\subsection{Discrete structural equations}

Through the same finite element procedures, the assembled algebraic equations for the structural response are

$$
\mathbf{M}_{s} \dot{\mathbf{v}}_{s}+\mathbf{C}_{s} \mathbf{v}_{s}+\mathbf{F}_{s}^{\text {int }}\left(\mathbf{u}_{s}\right)=\mathbf{F}_{s}
$$

where $\mathbf{v}_{s}$ is the velocity vector of the structural particles and $\mathbf{F}_{s}$ is the external load vector. The static resisting force vector, $\mathbf{F}_{s}^{i n t}$, is a nonlinear function of the nodal displacements $\mathbf{u}_{s}$, which are related to the velocities through time stepping methods. Finally, $\mathbf{M}_{s}$ and $\mathbf{C}_{s}$ are the structural mass and damping matrices.

\subsection{Discrete combined equations}

Particles connected to both the fluid and structural domains are identified as interface particles, whose contributions appear in both fluid and structural equations. From the structural system, the interface equations are extracted from Equation (20) and assigned additional $i$ and $s$ subscripts

$$
\begin{aligned}
& \mathbf{M}_{s s} \dot{\mathbf{v}}_{s}+\mathbf{M}_{s i} \dot{\mathbf{v}}_{i}+\mathbf{C}_{s s} \mathbf{v}_{s}+\mathbf{C}_{s i} \mathbf{v}_{i}+\mathbf{F}_{s}^{i n t}\left(\mathbf{u}_{s}, \mathbf{u}_{i}\right)=\mathbf{F}_{s} \\
& \mathbf{M}_{i s} \dot{\mathbf{v}}_{s}+\mathbf{M}_{i i}^{s} \dot{\mathbf{v}}_{i}+\mathbf{C}_{i s} \mathbf{v}_{s}+\mathbf{C}_{i i} \mathbf{v}_{i}+\mathbf{F}_{i}^{i n t}\left(\mathbf{u}_{s}, \mathbf{u}_{i}\right)=\mathbf{F}_{i}^{s}
\end{aligned}
$$

where $\mathbf{v}_{i}$ is the velocity vector of the interface particles. Similarly, the interface equations are extracted from Equations (13) and (15) for the fluid domain and given additional $i$ and $f$ subscripts

$$
\begin{gathered}
\mathbf{M}_{f f} \dot{\mathbf{v}}_{f}+\mathbf{K}_{f f} \mathbf{v}_{f}-\mathbf{G}_{f} \mathbf{p}=\mathbf{F}_{f} \\
\mathbf{M}_{i i}^{f} \dot{\mathbf{v}}_{i}+\mathbf{K}_{i i} \mathbf{v}_{i}-\mathbf{G}_{i} \mathbf{p}=\mathbf{F}_{i}^{f} \\
\mathbf{G}_{f}^{T} \mathbf{v}_{f}+\mathbf{G}_{i}^{T} \mathbf{v}_{i}+\mathbf{S p}=\mathbf{F}_{p}
\end{gathered}
$$

Equations (22) and (24) are combined in order to solve for the particle response on the fluid-structure interface.

\section{SOLUTION OF DISCRETIZED EQUATIONS}

Equations (21) and (22) are nonlinear equations because of the nonlinear term $\mathbf{F}_{s}^{\text {int }}\left(\mathbf{u}_{s}, \mathbf{u}_{i}\right)$ for the structural response. The fluid equations Equation (23) to Equation (25) are also nonlinear equations because of the geometric nonlinearity of fluid, for which all the matrices and vectors are computed at current configuration for every iteration. A time stepping method is applied to Equation (21) to Equation (25) in order to advance a simulation. The residual-based nonlinear solution algorithm is used to iteratively solve the equations. 


\subsection{Backward Euler time integration}

Backward Euler time integration is employed for the time stepping. The displacement and acceleration are expressed in terms of the velocity at the current time step and the displacement and velocity at the previous time step,

$$
\begin{aligned}
& \mathbf{u}_{n+1}=\mathbf{u}_{n}+\Delta t \mathbf{v}_{n+1} \\
& \dot{\mathbf{v}}_{n+1}=\frac{\mathbf{v}_{n+1}-\mathbf{v}_{n}}{\Delta t}
\end{aligned}
$$

The linearization of Equation (26) gives

$$
\begin{aligned}
d \mathbf{u}_{n+1} & =\Delta t d \mathbf{v}_{n+1} \\
d \dot{\mathbf{v}}_{n+1} & =\frac{1}{\Delta t} d \mathbf{v}_{n+1}
\end{aligned}
$$

which gives the update formula when the velocity increment is computed,

$$
\begin{aligned}
\mathbf{u}_{n+1}^{j+1} & =\mathbf{u}_{n+1}^{j}+\Delta t \Delta \mathbf{v}_{n+1} \\
\mathbf{v}_{n+1}^{j+1} & =\mathbf{v}_{n+1}^{j}+\Delta \mathbf{v}_{n+1} \\
\dot{\mathbf{v}}_{n+1}^{j+1} & =\dot{\mathbf{v}}_{n+1}^{j}+\frac{1}{\Delta t} \Delta \mathbf{v}_{n+1}
\end{aligned}
$$

where $n$ is the time step number and $j$ is the iteration number.

\subsection{Newton-Raphson algorithm}

The Newton-Raphson algorithm expresses equations in residual form

$$
\begin{aligned}
\mathbf{r}_{s}= & \mathbf{F}_{s}-\mathbf{M}_{s s} \dot{\mathbf{v}}_{s}-\mathbf{M}_{s i} \dot{\mathbf{v}}_{i}-\mathbf{C}_{s s} \mathbf{v}_{s}-\mathbf{C}_{s i} \mathbf{v}_{i}-\mathbf{F}_{s}^{i n t}\left(\mathbf{u}_{s}, \mathbf{u}_{i}\right) \\
\mathbf{r}_{i}= & \mathbf{F}_{i}^{s}+\mathbf{F}_{i}^{f}-\left(\mathbf{M}_{i i}^{f}+\mathbf{M}_{i i}^{s}\right) \dot{\mathbf{v}}_{i}-\left(\mathbf{C}_{i i}+\mathbf{K}_{i i}\right) \mathbf{v}_{i} \\
& -\mathbf{C}_{i s} \mathbf{v}_{s}-\mathbf{M}_{i s} \dot{\mathbf{v}}_{s}-\mathbf{F}_{i}^{i n t}\left(\mathbf{u}_{s}, \mathbf{u}_{i}\right)+\mathbf{G}_{i} \mathbf{p} \\
\mathbf{r}_{f}= & \mathbf{F}_{f}-\mathbf{M}_{f f} \dot{\mathbf{v}}_{f}-\mathbf{K}_{f f} \mathbf{v}_{f}+\mathbf{G}_{f} \mathbf{p} \\
\mathbf{r}_{p}= & \mathbf{F}_{p}-\mathbf{G}_{f}^{T} \mathbf{v}_{f}-\mathbf{G}_{i}^{T} \mathbf{v}_{i}-\mathbf{S p}
\end{aligned}
$$

For clarity, all velocities are collected in a single vector, $\mathbf{v}$, along with the vector, $\mathbf{r}$, of residual equations

$$
\mathbf{v}=\left[\begin{array}{c}
\dot{\mathbf{v}}_{s} \\
\dot{\mathbf{v}}_{i} \\
\dot{\mathbf{v}}_{f} \\
\mathbf{p}
\end{array}\right] \quad \mathbf{r}=\left[\begin{array}{c}
\mathbf{r}_{s} \\
\mathbf{r}_{i} \\
\mathbf{r}_{f} \\
\mathbf{r}_{p}
\end{array}\right]
$$

Following the procedure of Newton-Raphson algorithm, the derivative of the residual is taken with respect to the unknowns, then the velocity increment can be computed

$$
\begin{gathered}
\Delta \mathbf{v}^{j+1}=\left(\mathbf{K}_{T}^{j}\right)^{-1} \mathbf{r}^{j} \\
\mathbf{K}_{T}^{j}=-\frac{\partial \mathbf{r}}{\partial \mathbf{v}}=\left[\begin{array}{cccc}
\tilde{\mathbf{K}}_{T s s} & \tilde{\mathbf{K}}_{T s i} & \mathbf{0} & \mathbf{0} \\
\tilde{\mathbf{K}}_{T i s} & \tilde{\mathbf{K}}_{T i i} & \mathbf{0} & -\mathbf{G}_{i} \\
\mathbf{0} & \mathbf{0} & \tilde{\mathbf{K}}_{T f f} & -\mathbf{G}_{f} \\
\mathbf{0} & \mathbf{G}_{i}^{T} & \mathbf{G}_{f}^{T} & \mathbf{S}
\end{array}\right]
\end{gathered}
$$


Matrices with an over-tilde, for example, $\tilde{\mathbf{K}}_{T s s}$, are the algorithmic tangent matrices, that is, owing to the time integration method, for structural, interface, and fluid velocities. The matrix $\tilde{\mathbf{K}}_{T s s}$ is the tangent of structural residual to structural velocities,

$$
\tilde{\mathbf{K}}_{T s s}=\frac{\partial \mathbf{r}_{s}}{\partial \mathbf{u}_{s}}=\frac{1}{\Delta t} \mathbf{M}_{s s}+\mathbf{C}_{s s}+\Delta t \mathbf{K}_{T s s}
$$

where, $\mathbf{K}_{T s s}=\partial \mathbf{F}_{s}^{i n t}\left(\mathbf{u}_{s}, \mathbf{u}_{i}\right) / \partial \mathbf{u}_{s}$ is the tangent matrix of resisting force in structure to structural velocities. Analogous definitions for $\tilde{\mathbf{K}}_{T s i}$ and $\tilde{\mathbf{K}}_{T i s}$ are straightforward to obtain. The matrix $\tilde{\mathbf{K}}_{T f f}$ is the tangent of fluid residual to the fluid velocities,

$$
\tilde{\mathbf{K}}_{T f f}=\frac{\partial \mathbf{r}_{f}}{\partial \mathbf{u}_{f}}=\frac{1}{\Delta t} \mathbf{M}_{f f}+\mathbf{K}_{f f}
$$

where $\mathbf{K}_{f f}$ can be ignored for small $\Delta t$ or low fluid viscosity. The interface tangent matrix $\tilde{\mathbf{K}}_{T i i}$ has contributions from both fluid and structure,

$$
\tilde{\mathbf{K}}_{T i i}=\frac{\partial \mathbf{r}_{i}}{\partial \mathbf{u}_{i}}=\frac{1}{\Delta t}\left(\mathbf{M}_{i i}^{f}+\mathbf{M}_{i i}^{s}\right)+\left(\mathbf{C}_{i i}+\mathbf{K}_{i i}\right)+\Delta t \mathbf{K}_{T i i}
$$

where, $\mathbf{K}_{T i i}=\partial \mathbf{F}_{i}^{i n t}\left(\mathbf{u}_{s}, \mathbf{u}_{i}\right) / \partial \mathbf{u}_{i}$ is the tangent matrix of the structural resisting force to the interface velocities. Similar to the fluid tangent matrix in Equation (34), $\mathbf{K}_{i i}$ can be ignored for small $\Delta t$ or low fluid viscosity.

\section{FRACTIONAL STEP METHOD}

The monolithic matrix in Equation (32) is ill-conditioned due to coupling of the velocity and pressure fields, making it difficult to obtain a stable numerical solution for the incremental velocities and pressures. This is the reason that the FSM is utilized [6-9] in the PFEM. The FSM segregates the unknown pressures and velocities in to smaller systems of equations that are not ill-conditioned. Generally, the FSM can be summarized in three steps:

(1) Compute predictor velocities by ignoring pressure contributions arising from $\mathbf{G}_{i}$ and $\mathbf{G}_{f}$ in the first three rows of Equation (32);

(2) Solve for the pressures from the fourth row of Equation (32) using added or unadded, exact or approximated, mass and stiffness from the predicted velocities;

(3) Correct the velocities using the pressures found in step 2.

The FSM is applied in a mathematically exact way to the algebraic system of Equation (32). Using the first two rows of Equation (32),

$$
\left[\begin{array}{cc}
\tilde{\mathbf{K}}_{T s s} & \tilde{\mathbf{K}}_{T s i} \\
\tilde{\mathbf{K}}_{T i s} & \tilde{\mathbf{K}}_{T i i}
\end{array}\right]\left[\begin{array}{c}
\Delta \mathbf{v}_{s} \\
\Delta \mathbf{v}_{i}
\end{array}\right]-\left[\begin{array}{c}
\mathbf{0} \\
\mathbf{G}_{i}
\end{array}\right] \Delta \mathbf{p}=\left[\begin{array}{c}
\mathbf{r}_{s} \\
\mathbf{r}_{i}
\end{array}\right]
$$

the velocity increment for the structure and interface DOFs can be obtained

$$
\left[\begin{array}{c}
\Delta \mathbf{v}_{s} \\
\Delta \mathbf{v}_{i}
\end{array}\right]=\left[\begin{array}{cc}
\tilde{\mathbf{K}}_{T s s} & \tilde{\mathbf{K}}_{T s i} \\
\tilde{\mathbf{K}}_{T i s} & \tilde{\mathbf{K}}_{T i i}
\end{array}\right]^{-1}\left[\begin{array}{c}
\mathbf{r}_{s} \\
\mathbf{r}_{i}
\end{array}\right]+\left[\begin{array}{cc}
\tilde{\mathbf{K}}_{T s s} & \tilde{\mathbf{K}}_{T s i} \\
\tilde{\mathbf{K}}_{T i s} & \tilde{\mathbf{K}}_{T i i}
\end{array}\right]^{-1}\left[\begin{array}{c}
\mathbf{0} \\
\mathbf{G}_{i}
\end{array}\right] \Delta \mathbf{p}
$$

Similarly, using the third row of Equation (32)

$$
\tilde{\mathbf{K}}_{T f f} \Delta \mathbf{v}_{f}-\mathbf{G}_{f} \Delta \mathbf{p}=\mathbf{r}_{f}
$$


the incremental velocity for the fluid is

$$
\Delta \mathbf{v}_{f}=\tilde{\mathbf{K}}_{T f f}^{-1} \mathbf{r}_{f}+\tilde{\mathbf{K}}_{T f f}^{-1} \mathbf{G}_{f} \Delta \mathbf{p}
$$

Because of the assumption of low viscosity fluid, the $\tilde{\mathbf{K}}_{T f f}$ matrix is diagonal, making the incremental fluid velocity trivial to compute. On the other hand, the $\tilde{\mathbf{K}}_{T}$ matrices shown in Equation (37) are generally non-diagonal with unknown topology, making the solution for the incremental velocities of the structure and interface computationally intense.

In an attempt to reduce the computational cost of solving Equation (37), the matrix inverse can be written as

$$
\mathbf{B}=\left[\begin{array}{ll}
\mathbf{B}_{s s} & \mathbf{B}_{s i} \\
\mathbf{B}_{i s} & \mathbf{B}_{i i}
\end{array}\right] \equiv\left[\begin{array}{cc}
\tilde{\mathbf{K}}_{T s s} & \tilde{\mathbf{K}}_{T s i} \\
\tilde{\mathbf{K}}_{T i s} & \tilde{\mathbf{K}}_{T i i}
\end{array}\right]^{-1}
$$

where the blocks of the matrix $\mathbf{B}$ are the blocks of the matrix inverse, rather than the inverse of the corresponding blocks of $\tilde{\mathbf{K}}_{T}$. As shown in the following section, only selected entries from the $\mathbf{B}$ matrix are required to carry out the FSM in a computationally efficient manner.

The first step of the FSM is to compute the intermediate predictor velocities under the assumption that incremental pressures are zero. Using the B matrix defined in Equation (40), the predictor velocities are obtained by setting $\Delta \mathbf{p}$ to zero in Equations (37) and (39)

$$
\left[\begin{array}{c}
\Delta \mathbf{v}_{s}^{*} \\
\Delta \mathbf{v}_{i}^{*}
\end{array}\right]=\left[\begin{array}{ll}
\mathbf{B}_{s s} & \mathbf{B}_{s i} \\
\mathbf{B}_{i s} & \mathbf{B}_{i i}
\end{array}\right]\left[\begin{array}{l}
\mathbf{r}_{s} \\
\mathbf{r}_{i}
\end{array}\right]
$$

and

$$
\Delta \mathbf{v}_{f}^{*}=\tilde{\mathbf{K}}_{T f f}^{-1} \mathbf{r}_{f}
$$

The final velocities from Equations (37) and (39) can be substituted in to the fourth row of Equation (32), leading to the following system of equations for the incremental pressures

$$
\left(\mathbf{G}_{i}^{T} \mathbf{B}_{i i} \mathbf{G}_{i}+\mathbf{G}_{f}^{T} \tilde{\mathbf{K}}_{T f f}^{-1} \mathbf{G}_{f}+\mathbf{S}\right) \Delta \mathbf{p}=\mathbf{r}_{p}-\mathbf{G}_{i}^{T} \Delta \mathbf{v}_{i}^{*}-\mathbf{G}_{f}^{T} \Delta \mathbf{v}_{f}^{*}
$$

where $\mathbf{S}$ is the stabilization matrix. The term $\mathbf{G}_{i}^{T} \mathbf{B}_{i i} \mathbf{G}_{i}$ is related to the added-mass effect owing to both the mass and stiffness of the structure. For structures with high mass and/or stiffness, the components of the $\mathbf{B}$ matrix generally decrease in magnitude, making the added-mass effect negligible. Numerical experiments show, however, that retaining this term can lead to better convergence rates for a PFEM analysis. To circumvent the global-level computation of the added-mass effect, [9] approximated its effect using an interface Laplace matrix at the element level. The approach taken herein is to retain the term $\mathbf{G}_{i}^{T} \mathbf{B}_{i i} \mathbf{G}_{i}$ in its exact discrete form while obtaining the columns of $\mathbf{B}_{i i}$ from solutions to the following linear system of equations

$$
\left[\begin{array}{cc}
\tilde{\mathbf{K}}_{T s s} & \tilde{\mathbf{K}}_{T s i} \\
\tilde{\mathbf{K}}_{T i s} & \tilde{\mathbf{K}}_{T i i}
\end{array}\right]\left[\begin{array}{c}
\mathbf{x}_{s} \\
\mathbf{x}_{i}
\end{array}\right]=\mathbf{e}_{j}
$$

where $\mathbf{e}_{j}$ is a basis vector with $j^{t h}$ entry equal to one and all other entries equal to zero. The solution $\left[\begin{array}{ll}\mathbf{x}_{S} & \mathbf{x}_{i}\end{array}\right]^{T}$ is actually then the $j^{t h}$ column of the inverse matrix, B. Equation (44) would be solved $N$ times to obtain $\mathbf{B}_{s i}$ and $\mathbf{B}_{i i}$, where $N$ is the number of interface equations. For most FSI simulations, the size of the fluid-structure interface is a small portion of the total number of equations, which limits the computational cost of solving Equation (44) to only those DOFs along the interface.

The second term $\mathbf{G}_{f}^{T} \tilde{\mathbf{K}}_{T f f}^{-1} \mathbf{G}_{f}$ is the Discrete Laplace matrix, or PSC. In the classic FSM [6], this term was approximated by the Continuous Laplace matrix, which leads to mass loss on the free surface. Because $\tilde{\mathbf{K}}_{T f f}^{-1}$ is diagonal, computation of this term requires two matrix-matrix 
multiplications, and it can be performed exactly for the discrete system of equations, but with an additional computational cost compared with the classical FSM. The cost of finding $\mathbf{G}_{f}^{T} \tilde{\mathbf{K}}_{T f f}^{-1}$ is $O\left(n^{2}\right)$, as it is a scaling of the columns of $\mathbf{G}_{f}^{T}$ by the corresponding diagonal entries of $\tilde{\mathbf{K}}_{T f f}$, whereas the cost of $\left(\mathbf{G}_{f}^{T} \tilde{\mathbf{K}}_{T f f}\right) \mathbf{G}_{f}$ is $O\left(n^{3}\right)$. These costs can be reduced further by exploiting the topology of $\mathbf{G}_{f}$ via sparse matrix algorithms [17].

Using exact computations for the added-mass effect and the PSC, the solution of Equation (43) for the pressure increment leads to improved convergence rate and accuracy, as shown in the forthcoming examples. Once pressure is obtained, the incremental fluid velocities are corrected in order to satisfy the fluid incompressibility condition

$$
\Delta \mathbf{v}_{f}=\Delta \mathbf{v}_{f}^{*}+\tilde{\mathbf{K}}_{T f f}^{-1} \mathbf{G}_{f} \Delta \mathbf{p}
$$

Likewise, the incremental velocities of the structure and interface are corrected by

$$
\left[\begin{array}{c}
\Delta \mathbf{v}_{s} \\
\Delta \mathbf{v}_{i}
\end{array}\right]=\left[\begin{array}{c}
\Delta \mathbf{v}_{s}^{*} \\
\Delta \mathbf{v}_{i}^{*}
\end{array}\right]+\left[\begin{array}{cc}
\mathbf{B}_{s s} & \mathbf{B}_{s i} \\
\mathbf{B}_{i s} & \mathbf{B}_{i i}
\end{array}\right]\left[\begin{array}{c}
\mathbf{0} \\
\mathbf{G}_{i}
\end{array}\right] \Delta \mathbf{p}=\left[\begin{array}{c}
\Delta \mathbf{v}_{s}^{*} \\
\Delta \mathbf{v}_{i}^{*}
\end{array}\right]+\left[\begin{array}{c}
\mathbf{B}_{s i} \\
\mathbf{B}_{i i}
\end{array}\right] \mathbf{G}_{i} \Delta \mathbf{p}
$$

where the matrices $\mathbf{B}_{i i}$ and $\mathbf{B}_{s i}$ are found from the solution to Equation (44) in order to correct the structure and interface velocities. The correction of structural velocities by pressure in Equation (46) shows that this is a monolithic, rather than staggered, system.

In the real computation, further optimization can be performed. For example, if a direct solver is used, the factorization matrices can be stored, after the Equation (41) is solved. When Equation (44) is solved, only backward and forward substitution steps need to be performed, which are both of the order of $O\left(n^{2}\right)$. The total extra cost will be $O\left(n^{2}\right) \times N$, with $n$ the size of the equations and $N$ a small number compared with $n$. Whereas for an iterative solver, the term $\mathbf{G}_{i}^{T} \mathbf{B}_{i i} \mathbf{G}_{i}$ does not have to be constructed or stored. For the parallel computation environment, by using parallel direct solvers, such as MUMPS [18] and SuperLU [19], the factorization matrices may be stored in parallel, the columns of $\mathbf{B}_{i i}$ can be calculated in different processors simultaneously. For iterative solvers, they are good for parallelization in nature. Through various methods mentioned, the computational cost of the exact FSM on FSI can be reduced to a reasonable range, whereas the benefit is able to handle more complex Fluid-Structure Interaction problems with higher accuracy and stability.

\section{EXAMPLES}

The foregoing FSM method has been implemented in the OpenSees finite element software framework [20] as part of the framework's modules for PFEM analysis [21]. In each example, fluid response is simulated using a bubble function based MINI element [13], which satisfies the Inf-Sup condition via an internal cubic bubble node that is condensed at the element level.

Four verification tests (two pressure patch tests, one mass conservation test, and one test of the added-mass effect) are shown using the MINI fluid elements and various structural elements. These are followed by a validation test of a breaking dam on an elastic obstacle, a problem studied by many researchers. The final example demonstrates how the newly developed FSM handles structures characterized by nonlinear material response.

\subsection{FSI Patch test for pressures}

The execution of two patch tests developed by [22] shows that the newly developed FSM transmits constant stress boundary conditions to constant fluid pressures. An irregular mesh of fluid, solid, and beam elements comprise the first patch test shown in Figure 1. Constant stress boundary conditions of $P=10^{5} \mathrm{~Pa}$ are applied on the side and bottom of the patch while all other boundaries are fixed. The solid elements are linear-elastic with modulus $E=200 \mathrm{GPa}$ and Poisson ratio $v=0.4999$. The fluid elements haven densitivy $\rho=1000 \mathrm{~kg} / \mathrm{m}^{3}$ and visocity $\mu=0.001 \mathrm{~kg} / \mathrm{ms}$. Beam elements are used along the bottom of the patch in order to add normal traction to the fluid surface. The pressure contour in Figure 2 shows that an analysis using the new FSM leads to the exact solution of a constant pressure field in the fluid domain. 


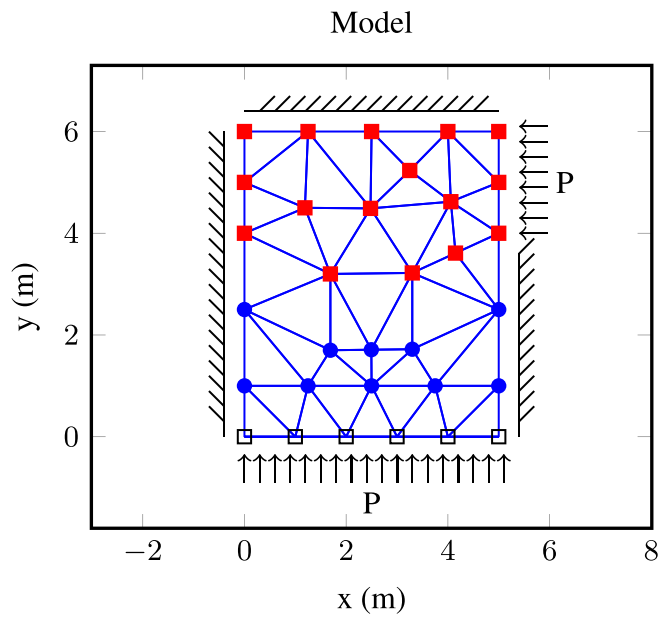

\section{- Fluid \\ - Solid \\ $\square$ Beam}

Figure 1. Fluid-structure interaction patch test \#1: solid and fluid meshes, and boundary conditions.

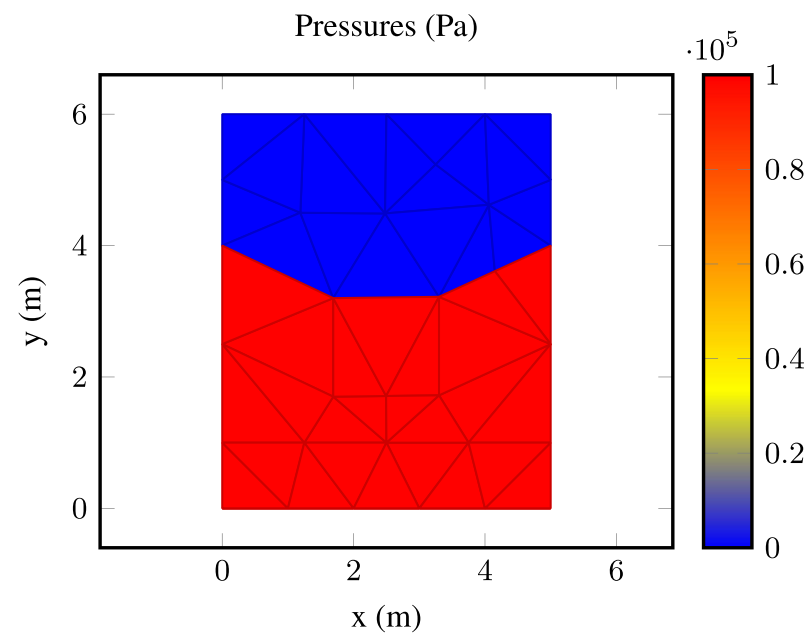

Figure 2. Fluid-structure interaction patch test \#1: pressure plot in the fluid domain.

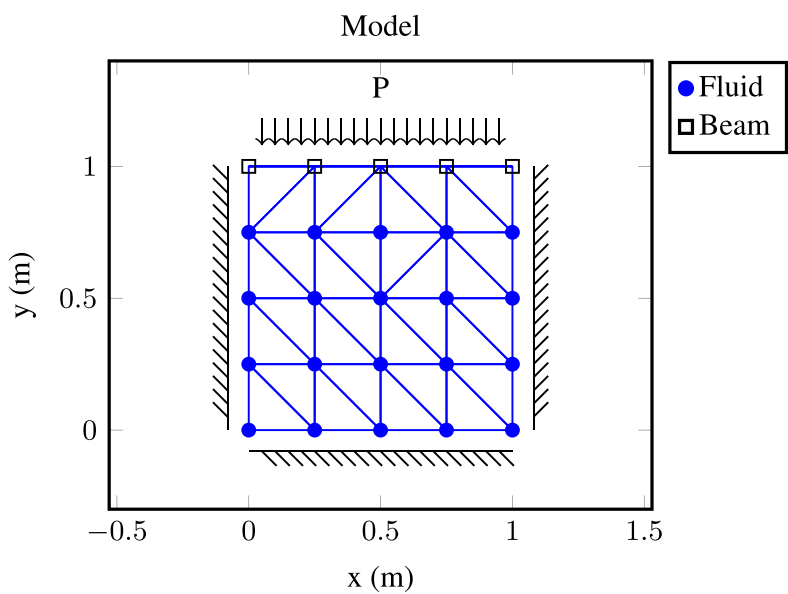

Figure 3. Fluid-structure interaction patch test \#2: solid and fluid meshes, and boundary conditions. 
In the second patch test, a regular mesh of fluid elements supports a line of displacement-based beam elements and is fixed along all other boundaries, as shown in Figure 3. The properties of the fluid and beam elements are the same as in the first patch test. A pressure load of $P=10^{5} \mathrm{~Pa}$ is applied on the beam. As in the previous patch test, analysis using the new FSM give the exact solution of constant pressure in the fluid, as shown in Figure 4.

\subsection{Mass conservation test}

For an incompressible flow, the incompressibility condition constrains the velocity field to be divergence free, resulting in conservation of the fluid mass. To ensure the corrected velocity is obtained, it is important to verify that mass is conserved. In this example, mass conservation of the newly developed FSM is compared with that for the classic FSM and FSMI [8].

Mass conservation is tested for viscous flow in a $1 \mathrm{~m}$ square container with five initial waves of height $0.1 \mathrm{~m}$, as shown in Figure 5. The fluid density and visocity are assumed to be $\rho=1000 \mathrm{~kg} / \mathrm{m}^{3}$ and $\mu=0.01 \mathrm{~kg} / \mathrm{ms}$, respectively. Slipping conditions are applied on the sides and bottom of the container. Although a seemingly simple example, this is a very difficult test for free-surface flow problems [8].

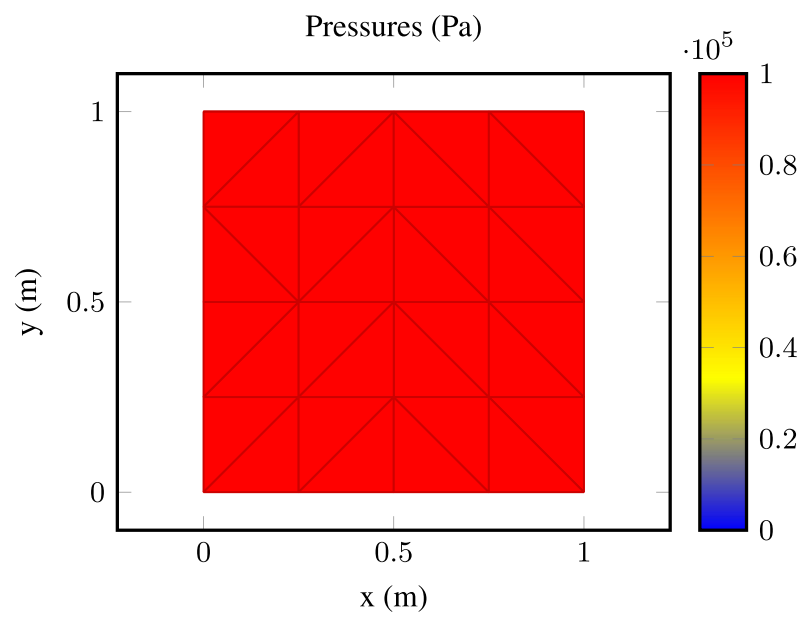

Figure 4. Fluid-structure interaction patch test \#2: pressure plot in the fluid domain.

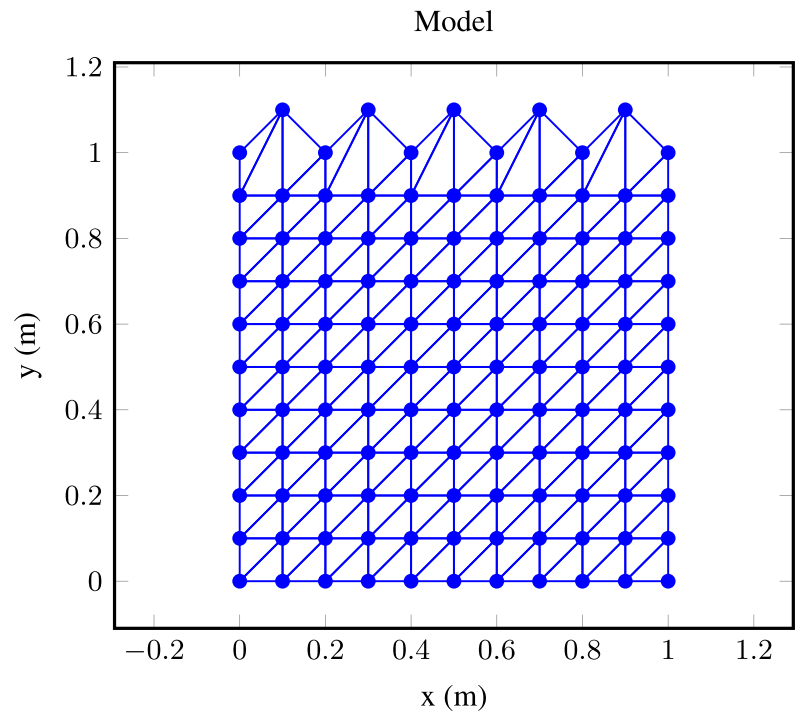

Figure 5. Small waves on the free surface for mass conservation test. 
The mass loss, computed as percentage change of volume, is shown in Figure 6(a) for the newly developed and classic FSM approaches. Mass loss is apparent for the classic FSM, where by $0.1 \mathrm{~s}$, the volume has increased by nearly $4 \%$. As shown in Figure 6(b), the volume decreases by about $0.012 \%$ when using the FSMI, whereas the newly developed FSM leads to a volume increase of $0.00081 \%$, two orders of magnitude less than the FSMI. Snapshots of the simulation using the newly developed FSM are shown in Figure 7 at different time steps and reveal the severe mesh distortions that make this a difficult verification test.

\subsection{Added-mass effect test}

The so-called added-mass effect refers to numerical instabilities that can occur in FSI problems where the fluid and structure have similar densities, and it can lead to unstable numerical solutions for a variety of staggered and monolithic numerical schemes for FSI. Elasticity of the solid and the simulation time step can also exacerbate the added-mass effect [9].

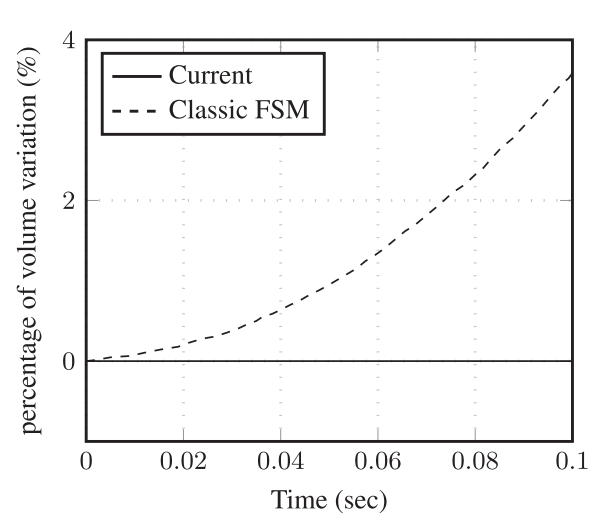

(a)

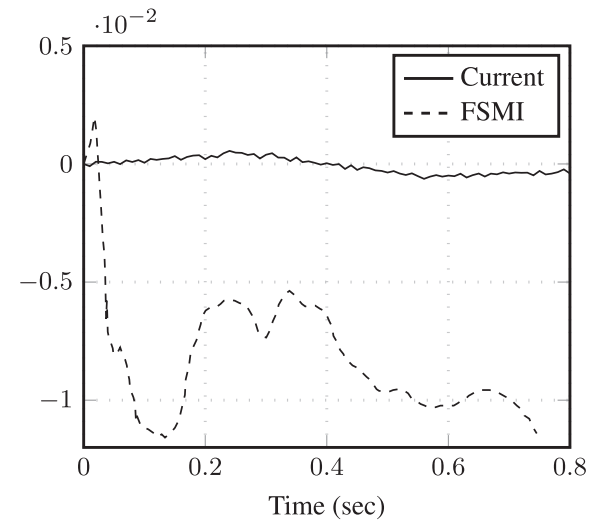

(b)

Figure 6. Comparison of total volume variation for mass conservation test.
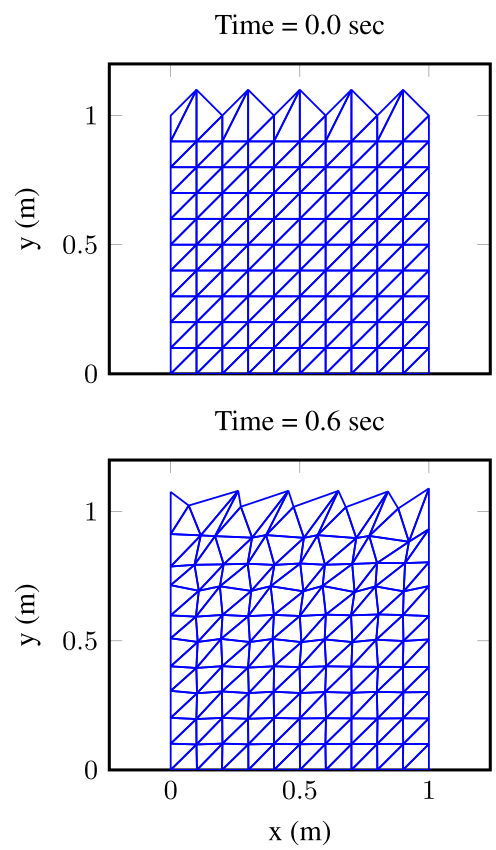

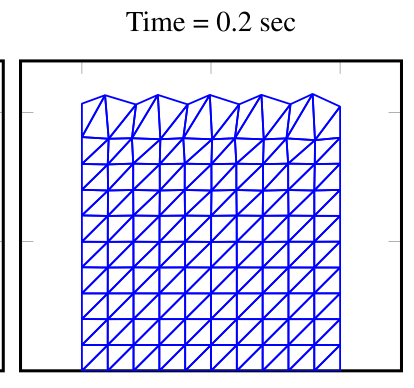

Time $=0.8 \mathrm{sec}$

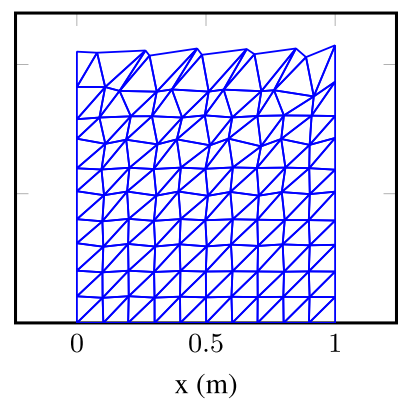

Time $=0.4 \mathrm{sec}$

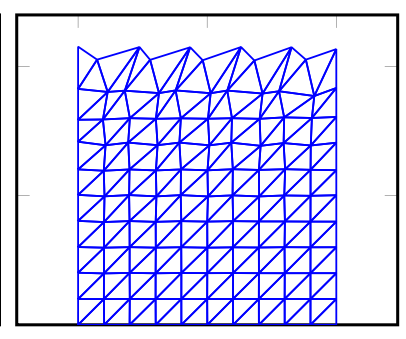

Time $=1.0 \mathrm{sec}$

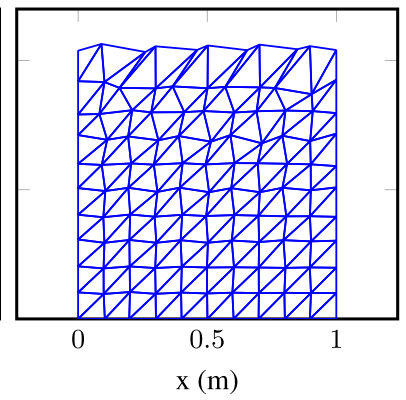

Figure 7. Snap shots for small waves on the free-surface at different time steps. 
An incompressible water column over an elastic solid, shown in Figure 8, is the model used to test the added-mass effect. The column width is $0.05 \mathrm{~m}$ and the heights of the fluid and solid columns are $0.75 \mathrm{~m}$ and $0.25 \mathrm{~m}$, respectively. The column walls are fixed in the horizontal direction, whereas the upper boundary is a free surface and the bottom boundary is fixed in both directions. The solid has density $\rho_{s}=1500 \mathrm{~kg} / \mathrm{m}^{3}$, elastic modulus, $E_{s}=2.3 \times 10^{5} \mathrm{~Pa}$, and Poisson ratio, $\nu=0.4$; while the fluid has density $\rho_{f}=1000 \mathrm{~kg} / \mathrm{m}^{3}$ and no viscosity. Vertical acceleration due to gravity is assumed to be $g=-10 \mathrm{~m} / \mathrm{s}^{2}$.

Using the newly developed FSM, the vertical displacement response, which is the same for all fluid particles, is shown in Figure 9(a). The period and magnitude of the response are similar to the results in [9] but are slightly smaller due to the exact added-mass and PSC terms used in this work. Figure 9(b) shows the vertical displacements for both the fluid and solid domains at different time steps, where each line is the profile of vertical displacements at a single time step. The perfect vertical segments in Figure 9(b) result from the incompressible condition of the water column.

To investigate how the newly developed FSM handles the added-mass effect, different values for the density, elastic modulus, and simulated time step are used and the resulting convergence behaviors are compared with those presented by [9]. A very strict tolerance on the velocity and pressure increments is used here with $\operatorname{tol}(\Delta v)=1 e^{-10}$ and $\operatorname{tol}(\Delta p)=1 e^{-10}$.

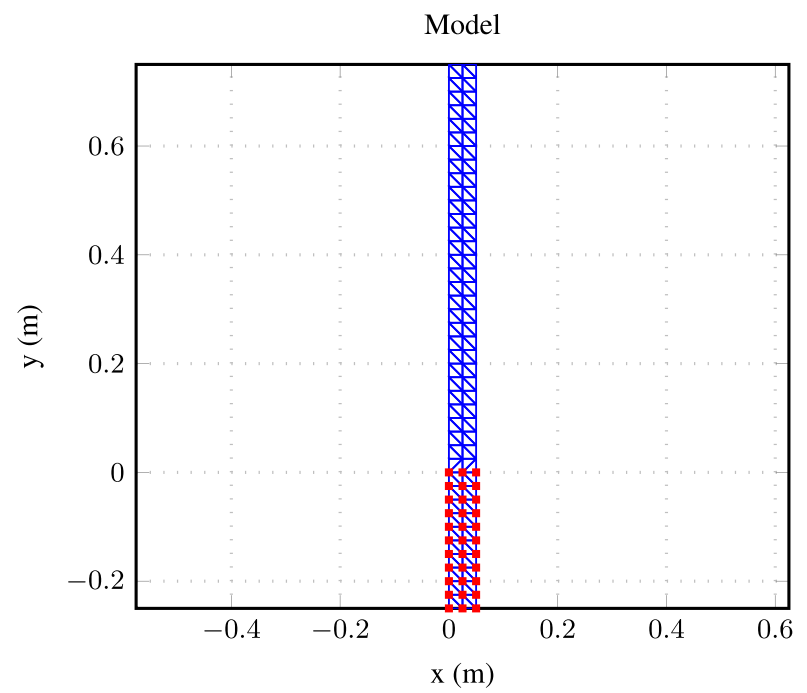

Figure 8. Water column over an elastic beam for added-mass effect test.

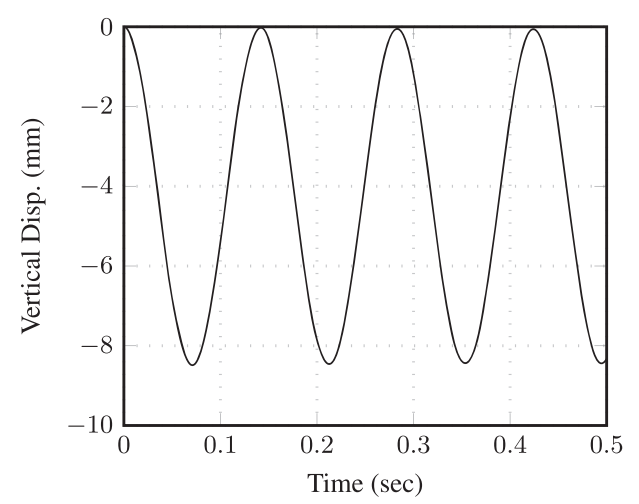

(a)

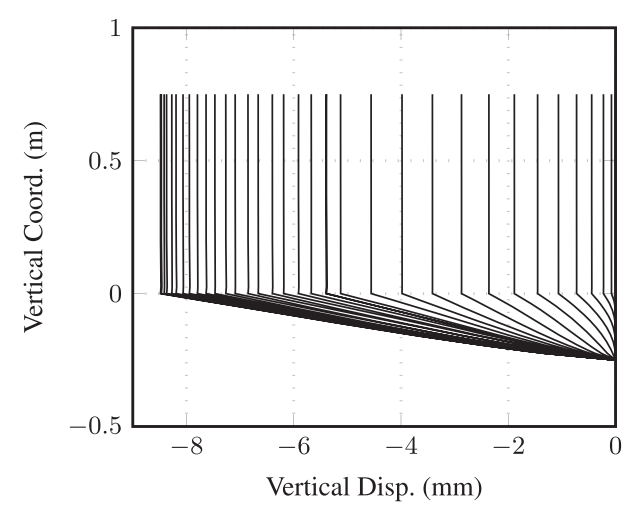

(b)

Figure 9. Vertical displacements for added-mass effect test: (a) time history for any fluid point. (b) Along the vertical direction for both domains at different time steps. 
Table I. Iterations to achieve convergence for different density ratio in added-mass effect test.

\begin{tabular}{lccl}
\hline$\frac{\rho_{s}}{\rho_{h}}$ & Current & $\beta \neq 0$ & \multicolumn{1}{c}{$\beta=0$} \\
\hline 10 & 3 iterations & 20 iterations & 20 iterations \\
7 & 3 iterations & 20 iterations & More than 40 iterations \\
6 & 3 iterations & 20 iterations & More than 40 iterations \\
5 & 3 iterations & 20 iterations & Does not converge \\
3 & 3 iterations & 19 iterations & Does not converge \\
1 & 3 iterations & 18 iterations & Does not converge \\
\hline
\end{tabular}

Table II. Iterations to achieve convergence for different Young's modulus in added-mass effect test.

\begin{tabular}{|c|c|c|c|}
\hline$E(\mathrm{~Pa}) ; \Delta t(\mathrm{~s})$ & Current & $\beta \neq 0$ & $\beta=0$ \\
\hline $2 \mathrm{e} 13 ; 0.2 \mathrm{e}-5$ & 3 iterations & 10 iterations & More than 40 iterations \\
\hline $2 \mathrm{e} 12 ; 0.5 \mathrm{e}-5$ & 3 iterations & 14 iterations & Does not converge \\
\hline $2 \mathrm{e} 11 ; 1 \mathrm{e}-5$ & 3 iterations & 18 iterations & Does not converge \\
\hline $2 \mathrm{e} 8 ; 1 \mathrm{e}-4$ & 4 iterations & 40 iterations & Does not converge \\
\hline $2 \mathrm{e} 7 ; 1 \mathrm{e}-3$ & 5 iterations & 36 iterations & Does not converge \\
\hline $2 \mathrm{e} 6 ; 1 \mathrm{e}-3$ & 5 iterations & 40 iterations & Does not converge \\
\hline $2 \mathrm{e} 6 ; 1 \mathrm{e}-2$ & 11 iterations & 34 iterations & 33 iterations \\
\hline $2 \mathrm{e} 5 ; 1 \mathrm{e}-2$ & 8 iterations & 36 iterations & Does not converge \\
\hline
\end{tabular}

Table III. Iterations to achieve convergence for different time steps in added-mass effect test.

\begin{tabular}{lccl}
\hline$\Delta t(\mathrm{~s})$ & Current & $\beta \neq 0$ & \multicolumn{1}{c}{$\beta=0$} \\
\hline $2 \mathrm{e}-5$ & 3 iterations & 23 iterations & 21 iterations \\
$1 \mathrm{e}-5$ & 3 iterations & 20 iterations & More than 40 iterations \\
$0.75 \mathrm{e}-5$ & 3 iterations & 18 iterations & Does not converge \\
$0.5 \mathrm{e}-5$ & 3 iterations & 16 iterations & Does not converge \\
$0.25 \mathrm{e}-5$ & 3 iterations & 11 iterations & Does not converge \\
\hline
\end{tabular}

For various ratios of solid to fluid density, Table I shows that current algorithm converges for all the cases in three iterations. This is in contrast with the 18 to 20 iterations required when approximating the added-mass effect with an interface Laplace matrix $(\beta \neq 0)$ and the slow (more than 40 iterations) or non-convergence when the added-mass effect is ignored $(\beta=0)$.

To investigate further the performance of the newly developed FSM for the added-mass effect, the same problem is run again with varying elastic modulus and simulation time steps, but equal density of the fluid and solid columns. Table II shows that using the exact approach to the addedmass effect leads to very fast convergence, with five or fewer iterations in all cases, save for those with the large time step of $0.01 \mathrm{sec}$. For the specific case of $E=2 e 11 \mathrm{~Pa}$ and solid to fluid density ratio equal to seven, the exact approach again is able to converge quickly (three iterations) for all time steps listed in Table III.

\subsection{Validation: deformation of an elastic plate subjected to water pressure}

This example compares simulations using the PFEM and newly developed FSM with experimental data and numerical results, obtained vis smoothed particle hydrodynamics, from a previous experiment of water pressure on an elastic gate [23]. The initial configuration is shown in Figure 10 with dimensions $A=0.1 \mathrm{~m}, L=0.079 \mathrm{~m}$, and $H=0.14 \mathrm{~m}$. The elastic gate is made of rubber, which is fixed at the upper end while its lower is end free and initially seals the container. The gate is modeled using a mesh of elastic beam elements with the corotational geometric transformation [24] to handle large displacements. The density of the gate is $\rho_{s}=1100 \mathrm{~kg} / \mathrm{m}^{3}$ and its elastic modulus 


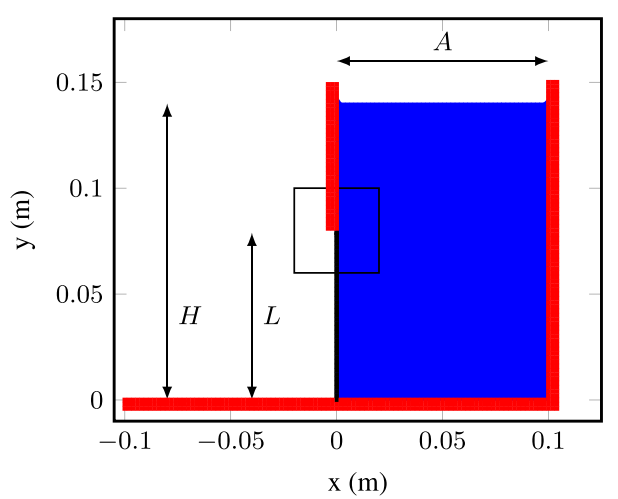

(a)

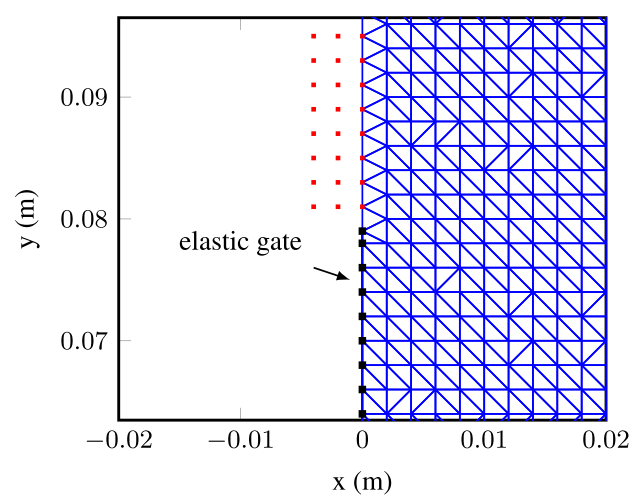

(b)

Figure 10. Initial configuration for the container with water and elastic gate.

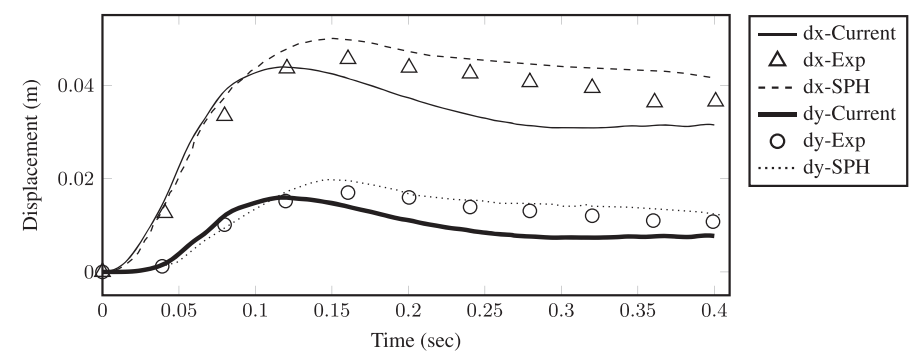

Figure 11. Comparison of the displacements of the free end of the elastic plate with experimental data and smoothed particle hydrodynamics approach.
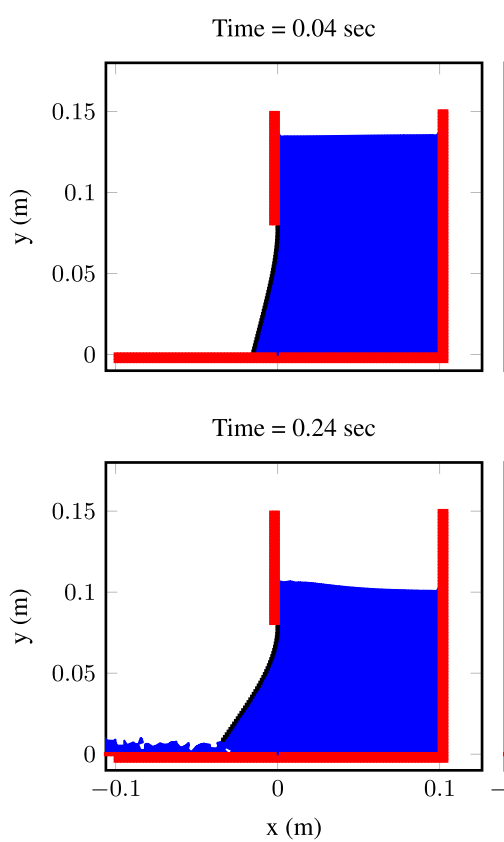

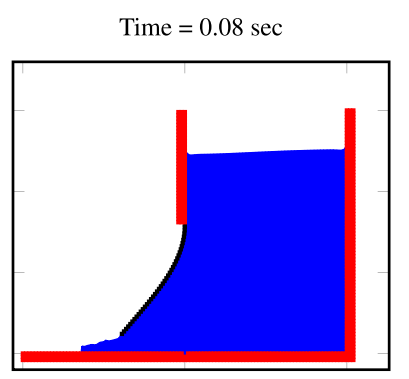

Time $=0.32 \mathrm{sec}$

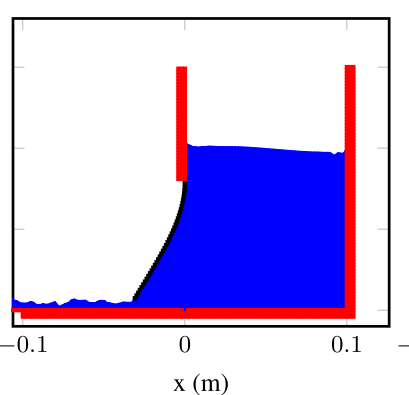

Time $=0.16 \mathrm{sec}$

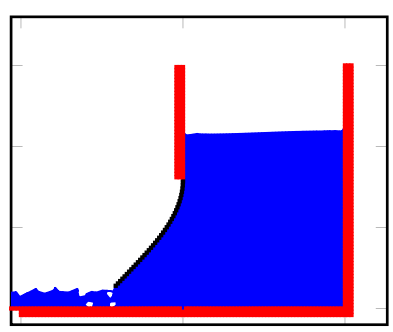

Time $=0.4 \mathrm{sec}$

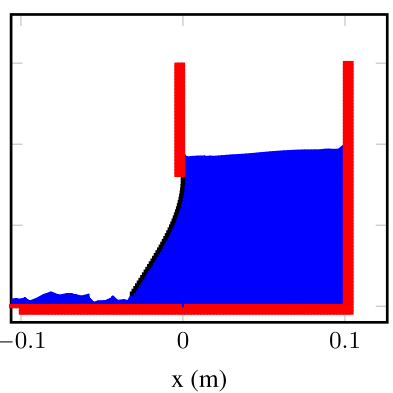

Figure 12. Snap shots of the deformation of elastic plate subjected to water pressure.

is assumed to be $E=10 \mathrm{MPa}$, although the true value of this property is uncertain and has been assumed to be as high as $14 \mathrm{MPa}$ in other replications of this experiment [25]. The fluid properties for density and viscosity are $\rho_{f}=1000 \mathrm{~kg} / \mathrm{m}^{3}$ and $\mu=0.001 \mathrm{~kg} / \mathrm{ms}$, respectively. 
The horizontal and vertical displacements of the free end of the gate are shown in Figure 11 and compared with the experimental results and simulations performed using smoothed particle hydrodynamics [23]. The agreement with experimental results is good considering the limitations of how the elastic gate is modelled using beam elements. Figure 12 shows snapshots of the simulation as the fluid reaches a steady state of flow out from behind the gate.

Due to the relatively large fluid-structure interface, this example provides an opportunity to assess the computational cost of the exact approach to handling the added-mass effect. As previously described and denoted in Equation (44), this approach requires the partial inverse of the structural and interface tangent stiffness matrices. In addition, this exact approach requires a global matrixmatrix multiplication of the discrete Laplace matrix, or PSC, as indicated in Equation (43). These two items represent the extra computational expense of the newly developed FSM compared with previous approaches that approximate the added-mass effect.

Table IV shows the CPU times required for the major operations required during one iteration within a simulation time step for the elastic gate problem. Three different mesh sizes for the fluid and gate are used in order to see how the operations scale with the increasing number of DOFs listed in Table IV. Note that the numbers of structural and interface DOFs increase linearly because the gate is discretized using line elements while the number of pressure DOFs increases quadratically. As shown in the table, the CPU time for the partial matrix inverse is very small compared with that required for matrix assembly and solving for pressure. This is due to the size of structure and interface being small compared with the size of the fluid domain; however, this is common for

Table IV. Top time consuming operations in one iteration with different mesh size for the deformation of the elastic gate.

\begin{tabular}{lrrr}
\hline mesh size (m) & 0.002 & 0.001 & 0.0005 \\
\hline \# of structural dofs & 42 & 81 & 160 \\
\# of interface dofs & 78 & 156 & 314 \\
\# of pressure dofs & 3623 & 14242 & 56482 \\
& \multicolumn{3}{c}{ CPU time (sec) } \\
& & & \\
Solving pressure & 0.57 & 7.22 & 84.66 \\
Assembling & 0.23 & 0.92 & 3.51 \\
Matrix multiplication & 0.01 & 0.04 & 0.18 \\
Partial inverse & $<0.01$ & 0.01 & 0.03 \\
\hline
\end{tabular}

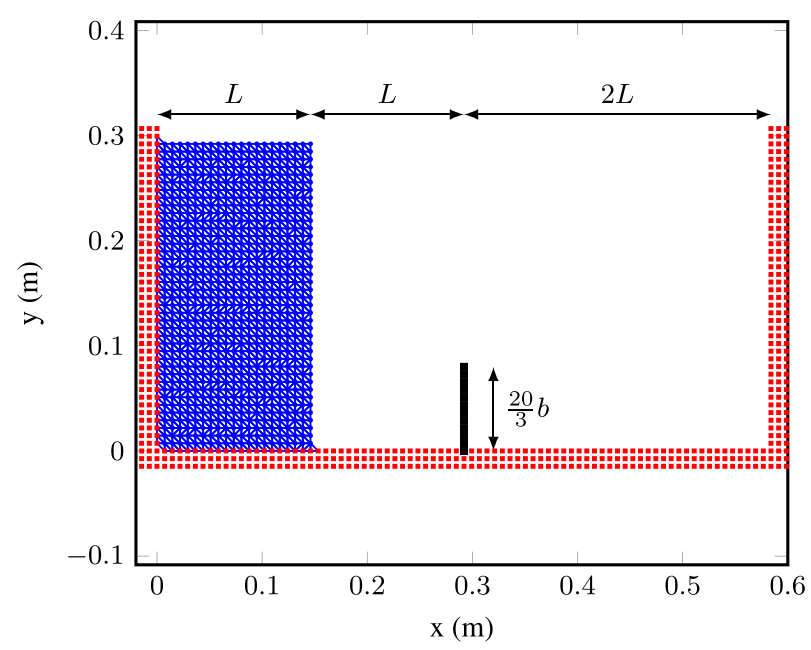

Figure 13. Breaking dam on elastic obstacle. 
realistic FSI simulation of coastal infrastructure where the structural model is small compared with the volume of fluid with which it interacts.

\subsection{Verification: breaking dam on elastic obstacle}

This example serves to verify the current FSM with results obtained using different FSI numerical methods. The model is of a dam break on an elastic obstacle fixed to be bottom of a tank, as shown in Figure 13. The characteristic length, $L$, is equal to $0.146 \mathrm{~m}$, and the elastic obstacle has a width of $b=0.012 \mathrm{~m}$ and height $20 \mathrm{~b} / 3=0.08 \mathrm{~m}$. As in the previous example, the elastic obstacle is modeled as a corotational mesh of beam elements having density $\rho_{s}=2500 \mathrm{~kg} / \mathrm{m}^{3}$, elastic modulus $E=10^{6} \mathrm{~Pa}$, and Poisson ratio $v=0$. The fluid density is $\rho_{f}=1000 \mathrm{~kg} / \mathrm{m}^{3}$ and its viscosity is $\mu=0.001 \mathrm{~kg} / \mathrm{ms}$.

Although experimental data is not available, this example has been studied by several researchers [25-28] and thus provides a good point of comparison for the newly developed FSM with previous simulation results. Snapshots of the dam break simulation using the newly developed FSM are shown in Figure 14. To highlight the final displaced shape of the elastic obstacle, the fluid mesh is not drawn in the final snapshot of Figure 14. Comparisons of the time history of horizontal tip displacement of the obstacle with simulations by the aforementioned researchers are shown in Figure 15 along the numerical method used by each researcher. The results of the current method agree well with the two previous PFEM formulations presented by [26] and [25], as shown in Figure 15(a) and (c), respectively.

\subsection{Demonstration: breaking dam on nonlinear material obstacle}

The final example demonstrates the ability of the newly developed FSM to simulate the interaction of fluid with a structure that responds in the nonlinear material range. The same breaking dam model is used with the obstacle comprised of a corotational mesh of displacement-based beam elements (constant axial deformation and linear curvature approximations). Two Gauss points are used in each element where cross-sections are discretized by ten fibers, each with the uniaxial bilinear steel stressstrain response shown in Figure 16(a). The stress-strain behavior is characterized by parameters of yield strength $F_{y}=5 e 4 \mathrm{~Pa}$, initial elastic tangent $E_{0}=10^{6} \mathrm{~Pa}$, and strain hardening ratio

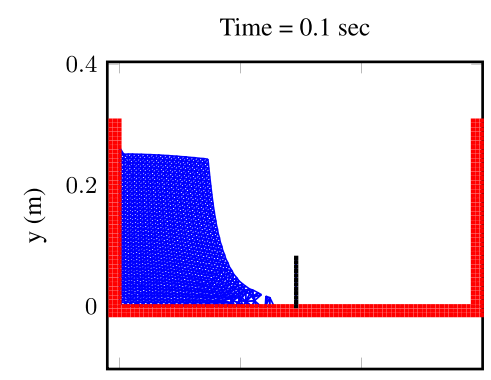

Time $=0.4 \mathrm{sec}$

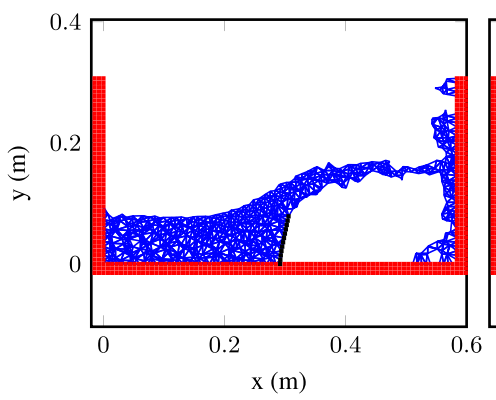

Time $=0.2 \mathrm{sec}$

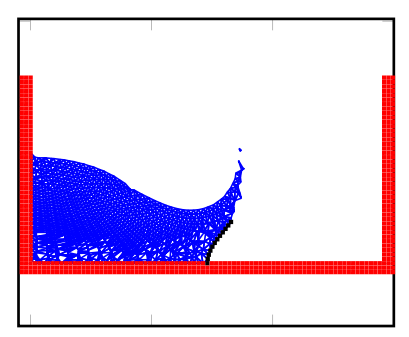

Time $=0.5 \mathrm{sec}$

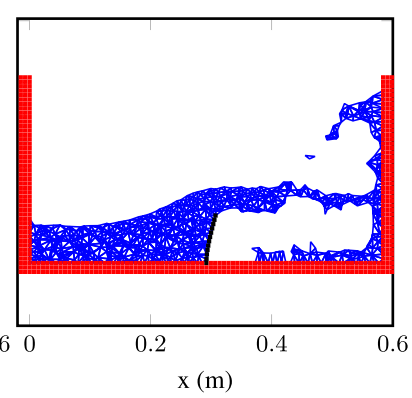

Time $=0.3 \mathrm{sec}$

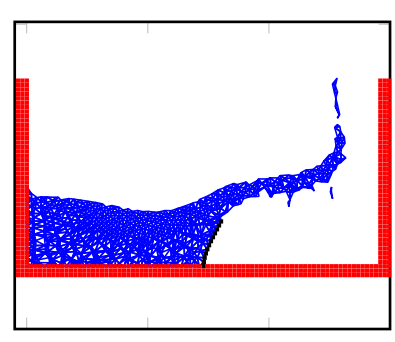

Time $=2.0 \mathrm{sec}$

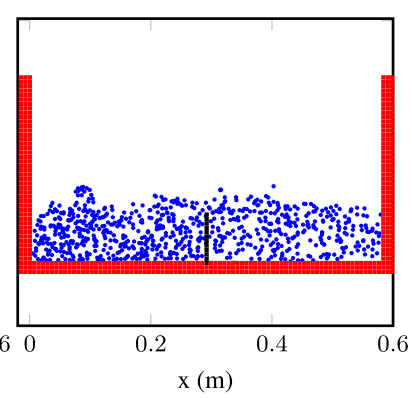

Figure 14. Snap shots for breaking dam on elastic obstacle at different time steps. 


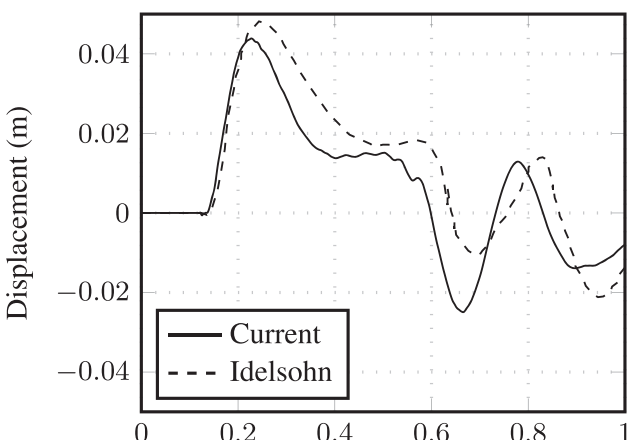

(a) Unified FSI formulation

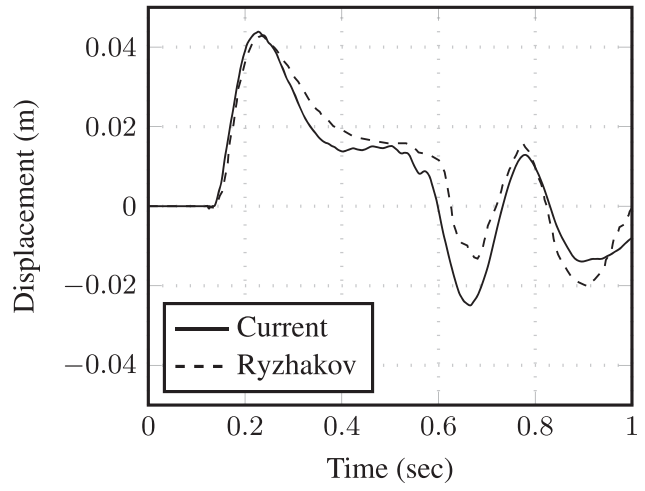

(c) Quasi-incompressible PFEM

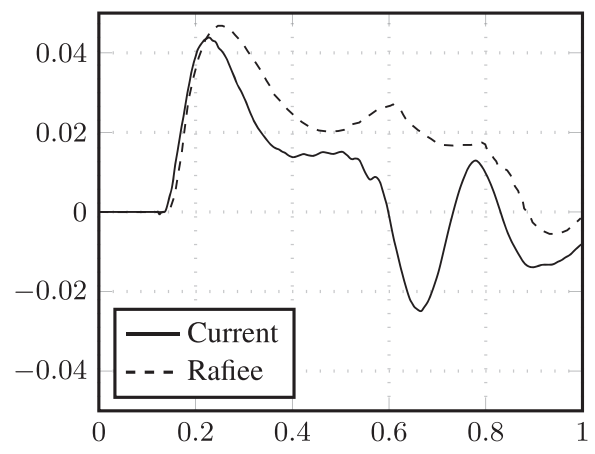

(b) SPH

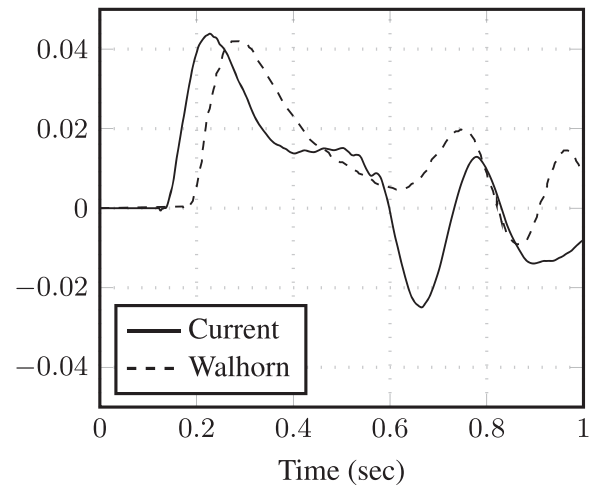

(d) Space-time FEM

Figure 15. Comparison of the displacements of the free end of the elastic obstacle with different numerical approaches.

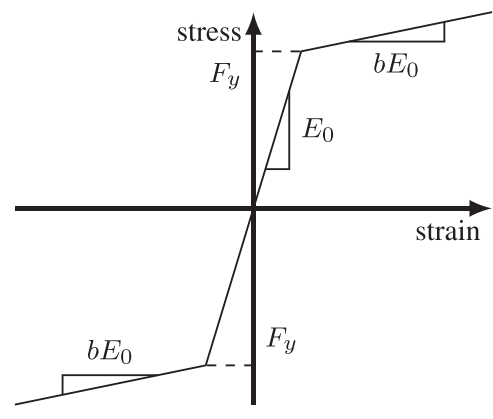

(a) stess-strain relationship

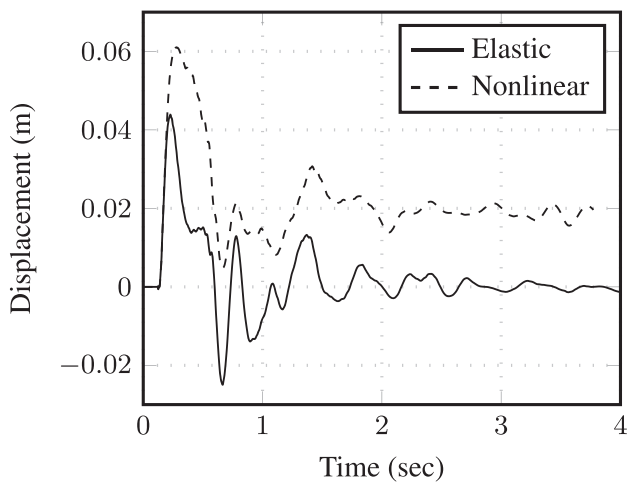

(b) Free end displacements

Figure 16. Nonlinear material for obstacle in dam break problem and comparison of free end displacements with elastic material.

$b=0.02$. The initial elastic tangent is the same as in the previous example in order to show the effects of nonlinear material response for the obstacle.

The time history of the obstacle's horizontal tip displacement is shown in Figure 16(b) along with snapshots of the simulation in Figure 17. Compared with the case of a linear-elastic obstacle, the nonlinear material shows larger peak displacement and permanent deformation, as expected. Note that the fluid mesh is not drawn in the final snapshot of Figure 17 in order to highlight the permanent deformation of the obstacle. 


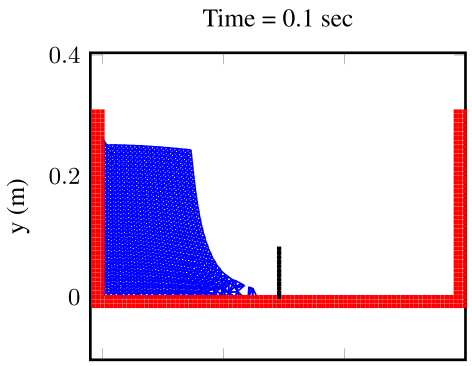

Time $=0.4 \mathrm{sec}$

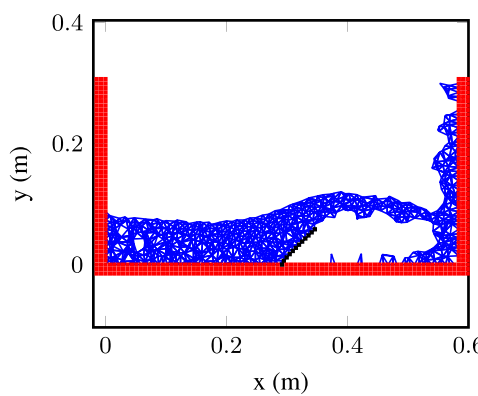

Time $=0.2 \mathrm{sec}$

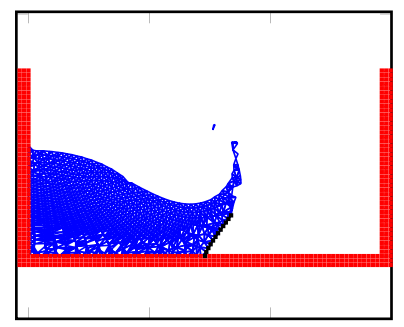

Time $=0.5 \mathrm{sec}$

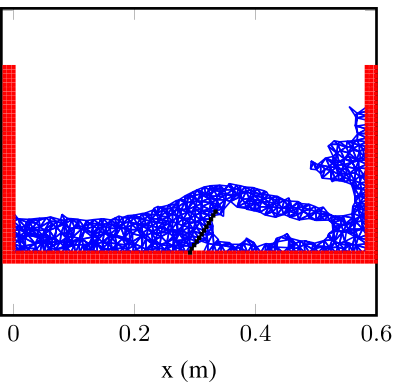

Time $=0.3 \mathrm{sec}$

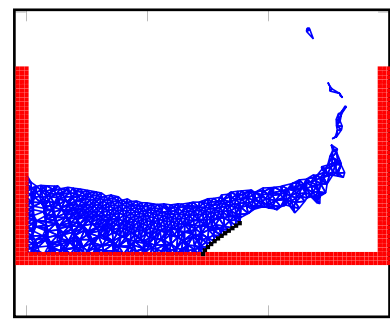

Time $=2.0 \mathrm{sec}$

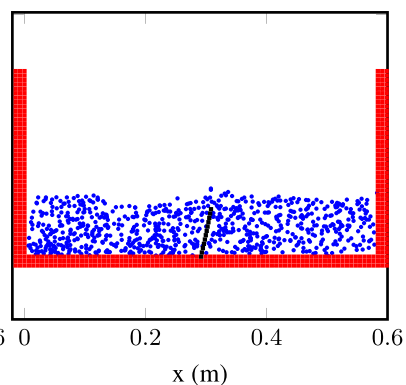

Figure 17. Snap shots for breaking dam on obstacle of nonlinear material at different time steps.

\section{CONCLUSIONS}

Handling added-mass terms with a mathematically exact approach in the FSM leads to improved numerical convergence without significant additional computational expense in FSI simulations using the PFEM. The additional computational expense depends on the size of the structure and the fluid-structure interface, which is generally very small relative to the size of the fluid domain for practical and realistic FSI simulations. Numerical examples show that pressure patch tests are satisfied, whereas the added-mass effect and mass loss on the free surface are both mitigated. In addition, verification and validation examples show that the exact method performs as well as previous incarnations of the FSM and is able to replicate physical experiments well. The ability to use arbitrary structural elements, including those that respond with material nonlinearity, is a significant advantage of this new FSM approach.

\section{ACKNOWLEDGEMENTS}

This material is based on work supported by the National Science Foundation under Grant No. 0847055. Any opinions, findings, and conclusions or recommendations expressed in this material are those of the authors and do not necessarily reflect the views of the National Science Foundation.

\section{REFERENCES}

1. Girault V, Raviart P. Finite Element Methods for Navier-Stokes Equations. Springer-Verlag: Berlin, 1986.

2. Gunzburger M. Finite Element Methods for Viscous Incompressible Flows. Academic Press: Boston, 1989.

3. Baiges J, Codina R. The fixed-mesh ale approach applied to solid mechanics and fluid-structure interaction problems. International Journal for Numerical Methods in Engineering 2010; 81:1529-1557.

4. Radovitzky R, Ortiz M. Lagrangian finite element analysis of newtonian fluid flows. International Journal for Numerical Methods in Engineering 1998; 43:607-619.

5. Tezduyar T, Mittal S, Ray S, Shih R. Incompressible flow computations with stabilized bilinear and linear equalorder-interpolation velocity-pressure elements. Computer Methods in Applied Mechanics and Engineering 1992; 95:221-242.

6. Oñate E, Idelsohn SR, Pin FD, Aubry R. The particle finite element method. an overview. International Journal of Computational Methods 2004; 1(2):267-307. 
7. Aubry R, Idelsohn SR, Oñate E. Fractional step like schemes for free surface problems with thermal coupling using the lagrangian pfem. Computational Mechanics 2006; 38:294-309.

8. Idelsohn SR, Oñate E. The challenge of mass conservation in the solution of free-surface flows with the fractionalstep method: Problems and solutions. International Journal for Numerical Methods in Biomedical Engineering 2010; 26:1313-1330.

9. Idelsohn SR, Pin FD, Rossi R, Oñate E. Fluid-structure interaction problems with strong added-mass effect. International Journal For Numerical Methods In Engineering 2009; 80:1261-1294.

10. Bathe KJ. Finite Element Procedures. Prentice-Hall: London, 1996.

11. Oñate E, García J, Idelsohn SR, Pin FD. Finite calculus formulations for finite element analysis of incompressible flows. eulerian, ale and lagrangian approaches. Computer Methods in Applied Mechanics and Engineering 2006; 195:3001-3037.

12. Cuvelier C, Segal A, van Steenhoven AA. Finite Element Methods and Navier-Stokes Equations. D. Reidel Publishing Company: Boston, 1986.

13. Arnold DN, Brezzi F, Fortin M. A stable finite element for the stokes equations. Mathematics And Statistics 1984; 21(4):337-344.

14. Bank RE, Welfert BD. A comparison between the mini-element and the petrov-galerkin formulations for the generalized stokes problem. Computer Methods in Applied Mechanics and Engineering 1990; 83(1):61-68.

15. Matsumoto J. A relationship between stabilized fem and bubble function element stabilization method with orthogonal basis for incompressible flows. Journal of Applied Mechanics 2005; 8:233-242.

16. Pierre R. Optimal selection of the bubble function in the stabilization of the p1-p1 element for the stokes problem. SIAM Journal on Numerical Analysis 1995; 32(4):1210-1224.

17. Davis T. Direct Methods for Sparse Linear Systems. SIAM: Philadelphia, 2006.

18. Amestoy P, Duff I, L'Excellent JY. Multifrontal parallel distributed symmetric and unsymmetric solvers. Computer Methods in Applied Mechanics and Engineering 2000; 184:501-520. MUMPS.

19. Li XS, Demmel JW. SuperLU_DIST: A scalable distributed-memory sparse direct solver for unsymmetric linear systems. ACM Transactions on Mathematical Software 2003June; 29(2):110-140.

20. McKenna F, Scott MH, Fenves GL. Nonlinear finite-element analysis software architecture using object composition. Journal of Computing in Civil Engineering 2010; 24(1):95-107.

21. Zhu M, Scott MH. Modeling fluid-structure interaction by the Particle Finite Element Method in OpenSees. Computers \& Structures 2014; 132:12-21. DOI: 10.1016/j.compstruc.2013.11.002.

22. Bathe K, Ledezma G. Benchmark problems for incompressible fluid flows with structural interactions. Computers \& Structures 2007; 85:628-644.

23. Antoci C, Gallati M, Sibilla S. Numerical simulation of fluid structure interaction by sph. Computers \& Structures 2007; 85(11-14):879-890.

24. Crisfield MA. Non-linear Finite Element Analysis of Solids and Structures, Vol. 1. John Wiley \& Sons: West Sussex, 1991.

25. Ryzhakov PB, Rossi R, Idelsohn SR, Oñate E. A monolithic lagrangian approach for fluid-structure interaction problems. Computational Mechanics 2010; 46:883-899.

26. Idelsohn SR, Marti J, Limache A, Oñate E. Unified lagrangian formulation for elastic solids and incompressible fluids: Application to fluid-structure interaction problems via the pfem. Computer Methods in Applied Mechanics and Engineering 2008; 197:1762-1776.

27. Rafiee A, Thiagarajan KP. An sph projection method for simulating fluid-hypoelastic structure interaction. Computer Methods in Applied Mechanics and Engineering 2009; 198(33-36):2785-2795.

28. Walhorn E, Kölke A, Hübner B, Dinkler D. Fluid structure coupling within a monolithic model involving free surface flows. Computers \& Structures 2005; 83(25-26):2100-2111. 
Copyright of International Journal for Numerical Methods in Engineering is the property of John Wiley \& Sons, Inc. and its content may not be copied or emailed to multiple sites or posted to a listserv without the copyright holder's express written permission. However, users may print, download, or email articles for individual use. 